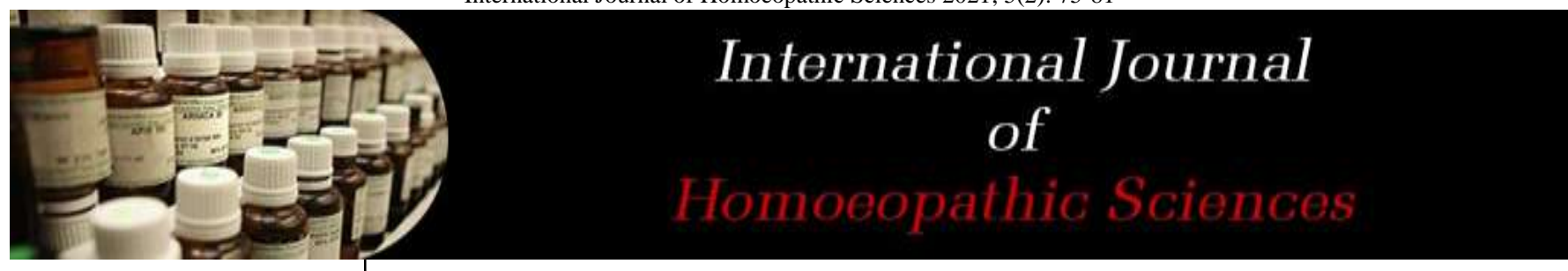

E-ISSN: 2616-4493 P-ISSN: 2616-4485 www.homoeopathicjournal.com IJHS 2021; 5(2): 75-81

Received: 05-01-2021

Accepted: 09-02-2021

Shreyank Kotian

BHMS, Intern, Father Muller

Homoeopathic Medical College and Hospital, Derlakatte,

Mangalore, Karnataka, India

Dr. Rajachandra G

Associate Professor,

Department of Practice of

Medicine, Father Muller

Homoeopathic Medical College

and Hospital, Derlakatte,

Mangalore, Karnataka, India

Corresponding Author:

Shreyank Kotian

BHMS, Intern, Father Muller

Homoeopathic Medical College

and Hospital, Derlakatte,

Mangalore, Karnataka, India

\section{Throwing light on sweet syndrome and its homoeopathic perspective}

\section{Shreyank Kotian and Rajachandra G}

DOI: $\underline{\text { https://doi.org/10.33545/26164485.2021.v5.i2b.364 }}$

\begin{abstract}
Sweet syndrome aka acute febrile neutrophilic dermatosis is one of the serious dermatological condition which goes undiagnosed due to several reasons. Unawareness of this condition may be one of the primary factors for missing the diagnosis which may lead to inaccurate treatment which may only lead to worsening the condition in a sick individual. Very few studies have been published in this area, hence the present investigator felt the need of reviewing few literatures and creating a material which will aid in early and accurate diagnosis of the condition. About 20 literature was reviewed based on the history, presentation, diagnosis and its probable homoeopathic treatment accordingly. All the information required for accurate diagnosis and its homeopathic approach was compiled into the present paper.
\end{abstract}

Keywords: Homoeopathy, sweet syndrome, acute febrile neutrophilic dermatosis, miasm, dermatology

\section{Introduction}

Homoeopathy has proved its efficacy time and again in various medical conditions, even when many systems of medicine have resorted to be of less advantageous in such conditions. The pathophysiology of the disease becomes the most important estimator for therapy in most other Medical systems but Homoeopathy is exclusively dependent on the symptoms so if a disease presents with symptoms then it can be managed with Homoeopathy.

There are certain problems existing in the medical science about certain diseases. Few of the diseases we do not know the exact aetiology, few we are not yet sure about the exact pathophysiology, few diseases do not have the sufficient tools to establish the diagnosis. But Homoeopathy do not suffer with such ambiguity as the mere presence of signs and symptoms will guide them to the therapeutics. This is the biggest advantage of the second largest medical system in the world, Homoeopathy. The Homoeopathic pathology i.e. the miasmatic evaluation would help us to identify a position for the current disease in the Hahnemann's classification of disease, which guides the physician in the treatment of that particular disease.

Homoeopathy has fashioned its scope in various dermatological conditions especially in all those diseases where the symptomatic presentation is clear. The gentle, harmless, permanent cure of these local manifestations according to the set of guidelines laid down by our Founder Dr. Samuel Hahnemann ${ }^{[1]}$ and other Stalwarts, where the malady can be removed without the use of external applications, thus making it the preferable mode of treatment for all. This review paper gives an insight about one of such rare dermatological conditions i.e. Sweet syndrome aka acute febrile neutrophilic dermatosis and its scope in homoeopathy.

\section{History}

In 1964, an English dermatologist named Dr. Robert D Sweet, described the disease ${ }^{[2]}$ that was observed in eight of his female patients. He described it as an illness with four distinct features
1. Fever
2. Acute, tender, red plaques
3. Leucocytosis
4. Papillary dermal infiltrate of neutrophils

This set of symptoms led to the nomenclature of the disease as 'Acute febrile neutrophilic dermatosis'. Dr. Sweet expected the descriptive terms to be the regular nomenclature 
of the condition. But' Sweet syndrome' ${ }^{[3]}$ ended up being the commonly used term in the fraternity. It is often confused with the illness to be related to disease caused by consumption sweet food items or Diabetes Mellitus due to its name.

In 1986, Dr. WP Su and Dr. HN Liu proposed a list of two major and four minor criteria for the diagnosis of Sweet Syndrome, but the modified and updated criteria given by Von Den Dreish P in 1994 is a generally accepted criteria among dermatologists.

\section{Incidence}

Sweet syndrome has shown a female predominance with the male female ratio $4: 1^{[4]}$.

This female predominance is seen only in the classical varieties of SS and not seen in Malignancy associated SS and Drug induced Sweet Syndrome.

Typically onset is seen between 30 to 60 years of age, but also seen in paediatric and geriatric ages also. There has been no observed racial predilection ${ }^{[5]}$.

In a study conducted by Hospach T, Von den Driesch P, Dannecker GE in $2009^{[6]}$, has shown that less than 70 children all over the world have reported with the diagnosis of Sweet syndrome out of which, only $42 \%$ had classical acute febrile neutrophilic dermatosis and the rest were associated with malignancies and drug induced cases. A need of further studies to identify the current prevalence/incidence of sweet syndrome is highly necessary for further studies on the condition.

\section{Etio-pathogenesis}

Genetic predisposition, especially seen in Japanese population, showed a predominance of the gene HLABw54 to be a risk factor in patients with Sweet syndrome ${ }^{[7]}$. Although the exact aetiologyis not identified till date, the various hypothesis' gives a narrower explanation on what causes the disease. There are currently two well explained theories.

\subsection{Sweet syndrome is a dermatological representation of classic immune complex vasculitis ${ }^{[8]}$}

Dr. Robert D Sweet, on careful histo-pathological analysis of cases with acute febrile neutrophilic dermatosis, consider the lack of vasculitis to be characteristic. But on more careful analysis of the histological sections of the classical SS cases, it showed that majority of cases have leukocytoclastic vasculitis

Although, in contrast, the presence of immunoglobulin and complement factors in direct immunofluorescence of the skin lesion, which is the hallmark of classic vasculitis, is absent in SS. Hence, even though the cases of classical SS showed leukocytoclastic vasculitis, there is no firm evidence to directly correlate Sweet Syndrome to be a representation of classical vasculitis.

4.3 A cascade of immunological events that is carried by T cells ${ }^{[8]}$

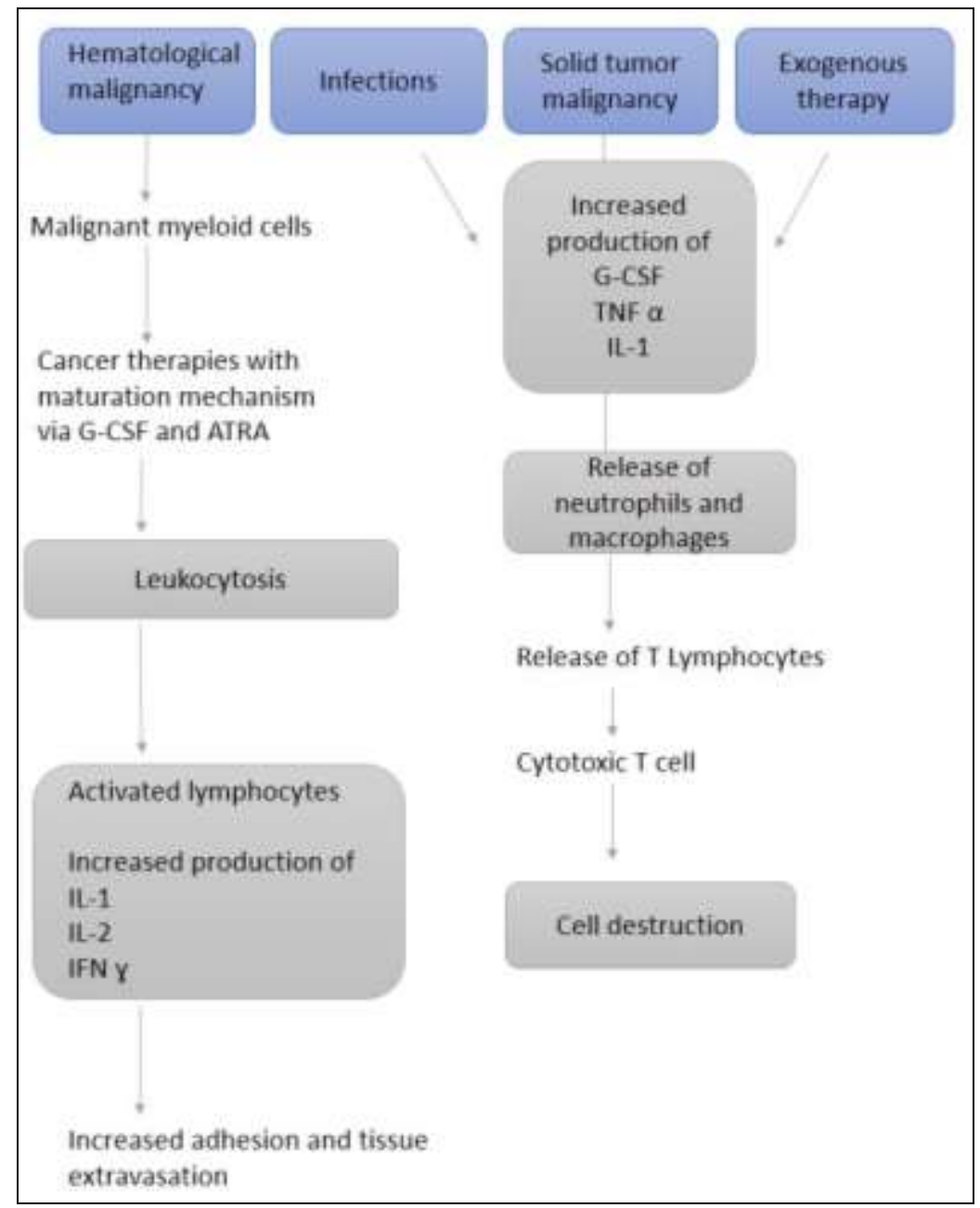

Fig 1: A cascade of immunological events that is carried by $\mathrm{T}$ cells 
Excessive infiltration of neutrophils into the skin (characteristic of Sweet Syndrome) will lead to dermal oedema. The continuous infiltration of these certain factors like (IL-1), IFN-y, IL-8, G-CSF and TNF explains the histopathological changes seen in SS.

Presence of all these factors, puts SS to close proximity to Pustular Psoriasis. T cell research on SS will give a better understanding of pathogenic process occurring in the cells of SS.

\section{Causes/risk factors ${ }^{[9]}$}

Sweet Syndrome is a relative phenomenon than an individual entity itself. Presence of classical presentation of Sweet Syndrome can be used as an indicative factor for the underlying disease. Some of the diseases which may have a role in pathogenesis of Sweet Syndrome are

\subsection{Infections}

- Upper respiratory infection, commonly Streptococcal infection (Important risk factor of SS)

- GIT infection - Yersinia

- Chlamydia

- Cytomegalovirus

- HIV

- Leprosy

- Tuberculosis

\subsection{Malignancies [20\% of Sweet Syndrome]}

- Acute Myeloid Leukaemia

- Solid tumours of Gastro Intestinal tract, Genitourinary Tract, Breasts

- Patients with older age, anaemia or thrombocytopenia, and without arthralgia are more likely to have malignancy-associated SS. Studies have recommended that patients with SS without clear aetiology should be followed up for at least 16 months to exclude a possible underlying haematological malignancy ${ }^{[10]}$.

\subsection{Drug induced}

a. G-CSF (Granulocyte Colony Stimulating factor)Hematopoietic drug substance used to treat AML)

b. Cotrimaxazole- the combination of trimethoprim (TMP) and sulfamethoxazole (SMX), used for the treatment of critically ill patients with infections caused by sensitive pathogens

c. Minocycline- effective against gram-positive andnegative infections.

d. Carbamazepine- anticonvulsants

e. Oral contraceptives

\subsection{Autoimmune disorders}

a. Systemic lupus erythematosus

b. Rheumatoid arthritis

c. Dermatomytosis

d. Hashimoto's thyroiditis

\subsection{Inflammatory bowel diseases}

a. Crohns disease

b. Ulcerative colitis

\subsection{Others}

a. Bechets disease

b. Erythema nodosum]

\section{Classification ${ }^{[11]}$}

Hence Sweet Syndrome is broadly classified based on the clinical setting in which Sweet Syndromeoccur

- Classical or idiopathic Sweet Syndrome

- Malignancy associated Sweet Syndrome

- Drug induced Sweet Syndrome

\subsection{Histiocytoid Sweet syndrome ${ }^{[12]}$}

This entity was first described by Requena et al. in 2005. It is considered as a variant of classic SS. It is histopathologically characterized by papillary dermal oedema with infiltration of histiocyte-like cells into the upper dermis. In this type the immature myeloid cells take the place of inflammatory cells which is usually seen in SS.

\section{Clinical features}

\subsection{Cutaneous manifestations}

- Abrupt/acute on set of

- Well demarcated lesions (Referred to as 'Relief of mountain range')

- Tender lesions

- Erythematous plaques, papules, nodes

- Pseudo vesiculosis

- Occasionally blisters that leads to annular slash aciform pattern

- Dermal oedema (Illusion of vesciculation) - Some lesions with dermal oedema gives a visual appearance as that of a vesicular lesion which may confuse the dermatologist to diagnose the condition clinically. Seen in the Histiocytoid variety of Sweet Syndrome

- Papules and plaques may combine to form large plaques up to $20 \mathrm{~cm}$ diameter

- In haemorrhagic malignancies, if local infection occurs, then atypical haemorrhagic bullae and ulceration may be formed.

- Ahn Et al- described variant of Xanthoma Like Lesions in Sweet Syndrome ${ }^{[12]}$.

Location of Lesions ${ }^{[8]}$

- Head

- Neck

- Legs (lower limb lesions similar to erythema nodosummorphologically and histologically)

- Arms (Particularly back of hands and fingers)

- Trunk is rarely involved

\subsection{Neutrophilic dermatosis of hands ${ }^{[14]}$}

It is a localized variant of Sweet syndrome seen on hands. It has a pustular appearance clinically and features of leukocytoclastic vasculitis histologically.

Hence it has been derived that neutrophilic dermatosis of hands are a variant of localised classical Sweet syndrome [13].

\section{After resolution of cutaneous manifestations}

- Persistent erythema may be present

- Scarring is rare

- Some reports of focal elastolysis at sites of previous inflammation was seen in children.

\subsection{Non cutaneous manifestations}

Febrile conditions

Fever, as mentioned in the nomenclature of the disease, is 
seen in more than $50 \%$ of patients with SS. This is usually due to the underlying infection or chronic inflammatory disease manifestation.

High fever maybe present before the onset of rash and may persist throughout the duration of illness ${ }^{[8]}$.

\subsection{Constitutional symptoms}

- Arthritis or Arthralgia is commonly seen among the individuals affected

- Myalgia maybe seen during or after fever

- Headache is also a recurring phenomenon in Sweet syndrome.

\subsection{Systemic complications}

- Occular-Conjunctivitis (38\%), Iritis, episcleritis, Limbal nodules

Sudden visual loss due to occlusive venous vasculitis [15]

Panuevitis with optic nerve involvement that resembles bechets disease ${ }^{[16]}$

- Liver- Transient liver enzymes elevation

- Oral- Apthous ulcers

- Lung- Aseptic pneumonia

- Children- Tibial pain

- Renal- Proteinurea followed by hematuria, redueced creatitine clearance, Acute renal failure

- CVS complications- Most dangerous complication in childhood (<3yrs of age). Occurs in $14 \%$ of those affected. Mortality rate of those with CVS complications is $40 \%$.

- CNS complications- Neuro-Sweet syndrome, a neurological complication of sweet syndrome, is seen mostly in Asians. Wide range of symptoms and signs can occur, depending on which part of the nervous system is affected. Usually presented with the SS typical skin lesions followed by neurological involvement or may even occur simultaneously. The most representative neurologic symptom that is seen is altered state of consciousness, followed by headache and memory disorders ${ }^{[17]}$.

\subsection{Histopathological features}

- Dense dermal infiltrate of neutrophils with leucocytoclasis, prominent papillary dermal oedema

- Focal neutrophil micro abscess formation maybe seen.

\section{Dreisch's criteria for diagnosis ${ }^{(18)}$}

\section{Major criteria}

- Abrupt onset of tender or painful erythematous plaques or nodules occasionally with vesicles, pustules or bullae.

- Predominantly neutrophilic infiltration of the dermis without obvious leukocytoclastic vasculitis.

\section{Minor criteria}

- Preceded by a nonspecific respiratory or gastrointestinal tract infection or vaccination or associated with inflammatory diseases, haemoproliferative disorders, solid malignant tumours, pregnancy.

- Accompanied by periods of general malaise and fever.

- Laboratory values during onset (3/4 necessary):

- ESR >20 mm

- C-reactive protein elevated

- Segmented nuclear neutrophils and stabs $>70 \%$ in peripheral blood smear

- Leucocytosis $>8000$.

- Excellent response to treatment with systemic corticosteroids or potassium iodide.

Two major and two minor criteria have to be present in order to diagnose the case as acute febrile neutrophilic dermatosis.

Drug-related Sweet syndrome: Diagnostic criteria ${ }^{[19]}$

A. Abrupt onset of painful erythematous plaques or nodules

B. Histopathological findings of dense neutrophilic infiltrate without evidence of leukocytoclastic vasculitis

C. Fever $>38^{\circ} \mathrm{C}$

D. Temporal relation between use of medication and clinical presentation or relapse with re-administration

E. Disappearance of lesions after drug discontinuation or treatment with systemic corticosteroids

\section{Prognosis}

- Depends on the severity of underlying or associated systemic disease

- Recurrences are not rare, and a chronic relapsing variant affects $15 \%$ of the patients

- In children, CVS complications have become associate with higher mortality rate ${ }^{[20]}$

\section{Homeopathic perspective}

Understanding the cause or the disease that is in the background becomes very necessary for homeopathic prescription for the condition. Hence in cases of Drug Induced or Malignancy Associated Sweet Syndrome, consideration of the background process should be definitely considered along with the scope of homoeopathy in those conditions, before treating

In cases of classical SS where no background processes are in action, the patients may be treated symptomatically.

The first thing that needs to be considered when approaching any condition is the Hahnemannian classification of the disease and the treatment protocol suggested to deal with that phenomenon.

Sweet syndrome maybe classified according to the hahnemann classification of diseases under the following headings.

1. Classical Sweet syndrome (Individual Acute disease)

2. Drug induced sweet syndrome (Artificial chronic disease)

3. Malignancy assoc. sweet syndrome and Idiopathic sweet syndrome (One sided disease)

\subsection{Sweet syndrome as an individual acute disease}

In Aphorism 5 [1], Hahnemann says "Useful to the physician in assisting him to cure are the particulars of the most probable exciting cause of the acute disease..." About acute diseases. As we already mentioned in the above sections of this article, there are multiple exciting causes which may lead to the formation of this entity. Here the treatment of the cause becomes the primary focus which may remove the symptoms of Sweet syndrome correspondingly.

HA Roberts said "In acute manifestations, the vital functions are often restored to complete harmony by and through their own power". Hence it is vital to understand that in mild cases, superficial treatment might be more 
harmful to the patient than it does any good.

The acute manifestations most of the times, if not always, are the transient explosion of the latent psora. Hence it is advised to treat with homeopathic remedies based on acute totality and then complete the cure/ avoid recurrence, if necessary, with an anti miasmatic remedy.

\subsection{Sweet syndrome as an artificial chronic disease}

This is the type of manifestation of Sweet syndrome which is due to the prolonged violent action of strong medication in its increasing doses. Because of the violent actions of these drugs, the vital force develops a reaction in the body to protect itself from the destructive primary action. According to Aphorism 75, these kind of diseases are the most incurable ones. This has to be remedied by the vital force itself. Identification of these factors and the corresponding action to avoid further progression becomes necessary before any therapeutic action is taken.

\subsection{Sweet syndrome as a one sided disease}

When we talk about the last variety, i.e. Malignancy associated Sweet Syndrome, it falls under the Hahnemannian classification of one sided diseases ${ }^{[1]}$, where the diseases has only few developed symptoms. Here we need to follow the criteria of treatment of one sided diseases according to Hahnemann. the treatment of Malignancy Associated Sweet syndrome maybe done on the basis of symptom similarity and correspondence i.e. by administration of the remedy which covers the similimum of the most prominent symptoms of the presentation. This can be done using proved symptoms of homoeopathic Materia Medica and with the aid of different repertories which guide us to the remedies of similar manifestation.

Of course, as Hahnemann suggested, we should focus on the accessory symptoms of the case available. If the remedy covers the uncommon peculiar symptoms, then cure may take place with the single remedy itself. If the selected remedy covers only the general symptoms and not the peculiar symptoms, then our totality should consist of both the accessory symptoms of medicine and disease. After the remedy administration, every time, a new totality will be formed based on the remaining symptoms and medicine may be prescribed. This process continues in succession until the cure is achieved.

The following are the remedies that is found in different repertories which may aid the homoeopathic doctor is finding the remedy when dealing with cases of Sweet Syndrome.

\subsubsection{Tender skin lesions}

\begin{tabular}{|c|c|c|}
\hline Kents repertory ${ }^{[21]}$ & BBCR $^{[22]}$ & $\begin{array}{c}\text { Boericke repertory } \\
{[23]}\end{array}$ \\
\hline & Arnica & Badiaga \\
Arnica & Bellaonno & Hepar \\
Belladonna & Phosphirc acid & Kali.C \\
Nux-Vomica & Asafoetida & Lachesis \\
Phosphoric Acid & Nux vomica & Oleander \\
Silicea & Sepia & Petroleum \\
Sulphur & Vertrum Alb & Psorinum \\
& Petroleum & Ther \\
& & Vinca \\
\hline
\end{tabular}

\subsubsection{Red Papular eruptions}

\begin{tabular}{|c|c|c|}
\hline Kents repertory ${ }^{[21]}$ & BBCR $^{[22]}$ & Boericke repertory $^{[23]}$ \\
\hline Sulphur & Mercurius & \\
Mercurious & Belladonna & \\
Kali-carb & Rhus-tox & Causticum \\
Calcarea carb & Phosphoric acid & Chamomilla \\
Phospherous & Graphitis & Graphitis \\
Kali-bich & Aconitum. & Lycopodium \\
Ammonium carb & Sepia & Mercurious \\
Sepia & Dulcamara & Petroleum \\
Petroleum & Pulsatilla & Sulphur \\
Kali-iod & Ant-crud & \\
Sul-acid & Causticum & \\
Mezereum & & \\
\hline
\end{tabular}

\subsubsection{Dermal oedema}

\begin{tabular}{|c|c|c|}
\hline Kents repertory ${ }^{[21]}$ & BBCR $^{[22]}$ & Boericke repertory [23] \\
\hline Mercurius & Rhus-tox & Acet.acid \\
Rhus-t.ox & Mercurius & Agaricus \\
Sulphur & Arsenic & Anacardium \\
Sepia & Sulphur & Apis Mellifica \\
Sambucus & Sambucus & Arsenic \\
Belladonna & Belladonna & Digitalis \\
Pulsatilla & Lycopodium & Helleborous \\
Kali-carb & Pulsatilla & Prunus sp \\
Thuja & Kali-carb & Rhus tox \\
& & Thyrodinum \\
\hline
\end{tabular}

10.3.4 Fever eruptions

\begin{tabular}{|c|c|c|}
\hline Kents repertory [21] & BBCR [22] & Boericke repertory ${ }^{[23]}$ \\
\hline & Mercurius & \\
Ignitia & Am-carb & Euphrasia \\
Apis Mellifica & Belladonna & Gelsemium \\
Rhus-tox & Crocus Sativus & Kali bich \\
Copaiva & Arsenic & Aconite \\
Sulphur & Bryonia & Pulsatilla \\
Rhus-v. & Sulphur & Sticta. \\
& Hyosiamus & \\
& Stramonium & \\
\hline
\end{tabular}




\subsubsection{Neutrophilic dermatosis of hands (Based on Kent's repertory)}

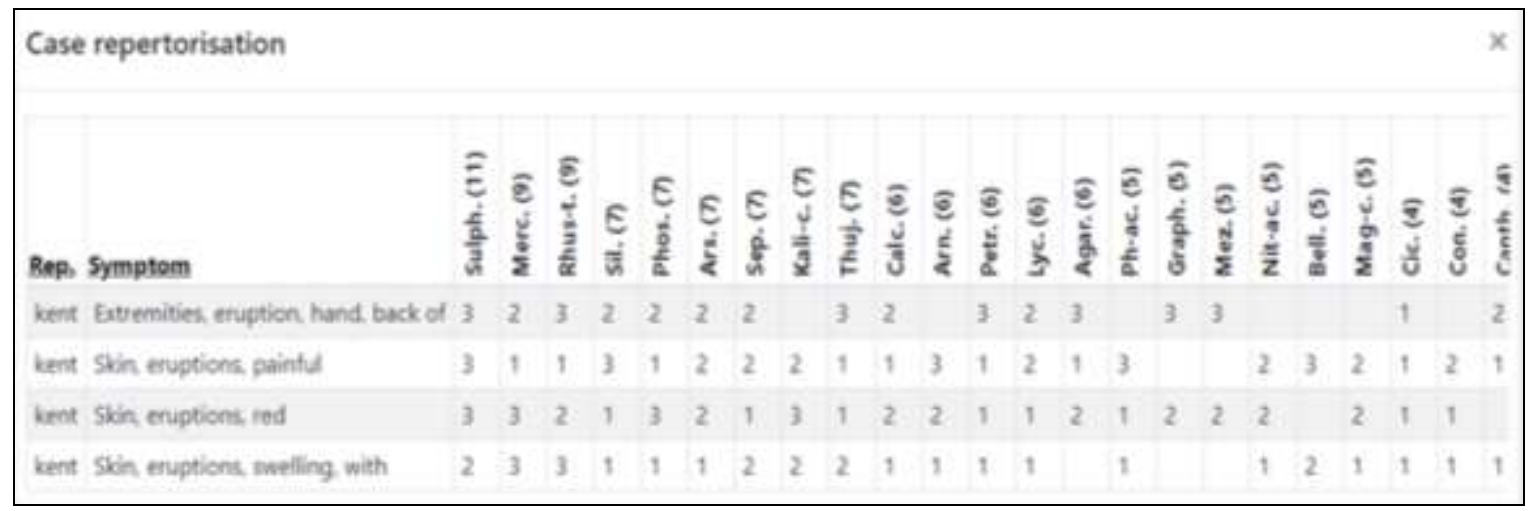

Fig 2: Neutrophilic dermatosis of hands (Based on Kent's repertory)

\subsubsection{Specific remedies from herring guiding symptoms} $[24,25]$

- Skin eruptions, nodular, painful- Natrum mur

- Skin eruptions, pustular painful- Tarentula

- Skin subcutaneous swelling- Kali-mur

- Skin, Eruption, Pustules, Malignant, Pyaemia, hectic fever- Crotalus horridus

- Skin, Inflammation (dermatitis), Pressure, tender on Arnica

\subsection{Miasmatic understanding of sweet syndrome}

The Miasmatic diagnosis of any condition will depend on the stage of the disease and its underlying cause for most of its spectrum.

In Sweet syndrome, when considering the Malignancy Associated SS, it will be predominantly under the sycosis or syphilis miasm (proliferative/destructive nature) ${ }^{[26]}$, with respect to the grading and progression of the underlying malignancy, and rarely seen under Psoric miasm

Although, when considering the skin symptoms alone, in cases of Classical acute febrile neutrophilic dermatosis, the following miasms are involved ${ }^{[27]}$

- Rawness or soreness of any skin eruptions - Syphillitic

- Red and haemorrhagic skin lesions- Tubercular

- Eruptions after vaccinations (drug induced)- Sycotic

- Exhaustion and general symptoms with Skin eruptionsTubercular

- Nodular swelling - Sycotic

- Papular eruptions- Psoric

- Painful boils- Psora

- Scars after tumours- Sycotic

\section{Conclusion}

The scope of homoeopathy in dermatological conditions is not to be questioned after many evidence based studies done over the years. But the preparedness of any homoeopath to treat rare conditions is always $\mathrm{s}$ necessary, especially considering the scope of thousands of remedies at our disposal. Understanding the mental cause, the maintaining factors of mind and body, and also the thorough understanding of the miasmatic diagnosis and treatment will help us encounter any rare conditions that come to us.

Further specific case studies or research studies of Homoeopathy on such rare skin conditions will help us improve the scope and create a mark in treatment of dermatological conditions with Homeopathy

\section{References}

1. Hahnemann S. Organon of medicine. Boericke \& Tafel 1912, 186-195.

2. Sweet RB. An acute febrile neutrophtlic dermatosts. British Journal of Dermatology 1964;76(8-9):349-56.

3. Sweet RD. Acute febrile neutrophilic dermatosis-1978. British Journal of Dermatology 1979;100(1):93-9.

4. Webb K, Hlela C, Jordaan HF, Suliman S, Scriba T, Lipsker D, Scott C. A review and proposed approach to the neutrophilic dermatoses of childhood. Pediatric Dermatology 2015;32(4):437-46.

5. Cohen PR. Sweet Syndrome [Internet]. Rare Disease Database 2020. https://rarediseases.org/rarediseases/sweet-syndrome/

6. Hospach T, Von den Driesch P, Dannecker GE. Acute febrile neutrophilic dermatosis (Sweet's syndrome) in childhood and adolescence: two new patients and review of the literature on associated diseases. European journal of pediatrics 2009;168(1):1-9.

7. Hisanaga K, Iwasaki Y, Itoyama Y. Neuro-Sweet Disease Study Group. Neuro-Sweet disease: clinical manifestations and criteria for diagnosis. Neurology 2005;64(10):1756-61.

8. Harper J, Oranje AP. Harper's Textbook of pediatric dermatology. John Wiley \& Sons 2019.

9. Valia RG, Valia AR. IADVL Textbook of dermatology. Bhiani publication house 2008.

10. Marcoval J, Martín-Callizo C, Valentí-Medina F, Bonfill-Ortí M, Martínez-Molina L. Sweet syndrome: long-term follow-up of 138 patients. Clinical and experimental dermatology 2016;41(7):741-6.

11. Habif TP. Clinical Dermatology E-Book. Elsevier Health Sciences 2015.

12. Huang CF, Wu BY, Liaw FY, Wang WM, Chiang CP. Histiocytoid Sweet syndrome: report of two cases and review of the literature. Dermatologica Sinica 2012;30(2):71-4.

13. Ahn SJ, Choi JH, Sung KJ, Moon KC, Koh JK. Sweet's syndrome presenting with lesions resembling eruptive xanthoma. British Journal of Dermatology 2000;143(2):449-50.

14. Walling HW, Snipes CJ, Gerami P, Piette WW, Reddy ES, Sharma PK, Raj PP. A clinical study on effect of Plantago in gingivitis by assessing bleeding and plaque index. Indian Journal of Research in Homoeopathy 2018;12 (3):132, 142(1):57-63.

15. Song WK, Bang D, Choi YJ, Lee SC, Oh SH. Sudden 
visual loss due to occlusive venous vasculitis associated with Sweet syndrome. Archives of dermatology 2009; 145(2):216-8.

16. Matsumiya W, Kusuhara S, Yamada Y, Azumi A, Negi A. Sweet's syndrome with panuveitis resembling Behcet's disease. Japanese journal of ophthalmology 2012;56(3):268-72.

17. Drago F, Ciccarese G, Agnoletti AF, Sarocchi F, Parodi A. Neuro sweet syndrome: a systematic review. A rare complication of Sweet syndrome. Acta Neurologica Belgica 2017;117(1):33-42.

18. Von den Driesch P. Sweet's syndrome (acute febrile neutrophilic dermatosis). J Am Acad Dermatol 1994;31:535-56.

19. Villarreal-Villarreal CD, Ocampo-Candiani J, Villarreal-Martínez A. Sweet syndrome: a review and update. Actas Dermo-Sifiliográficas (English Edition) 2016;107(5):369-78.

20. Halpern J, Salim A. Pediatric sweet syndrome: case report and literature review. Pediatric dermatology 2009;26(4):452-7.

21. Kent JT. Repertory of the homoeopathic materia medica. B. Jain Publishers 1992.

22. Boger CM. Boenninghausen's Characteristics. Jamadade AK, Adi BS, Reddy ES. single blind randomized control study on the efficacy of homoeopathic management in coronary artery disease. International journal of alternative and complementary medicine 2020;26:41-5. Materia Medica and Repertory 1905.

23. Boericke W. Pocket Manual of Homoeopathic Materia Medica \& Repertory: Comprising of the Characteristic and Guiding Symptoms of All Remedies (clinical and Pahtogenetic [sic]) Including Indian Drugs. B. Jain publishers 2002.

24. Knerr CB, Hering C. A Repertory of Hering's Guiding Symptoms of our Materia Medica. FA Davis 1896.

25. Hering $\mathrm{C}$. The guiding symptoms of our materia medica. Stoddart 1890.

26. Hahnemann S. The chronic diseases. Boericke \& Tafel 1904.

27. Banerjea SK. Miasmatic Prescribing. B Jain Pub Pvt Limited 2007. 